\title{
Melodinhenine B attenuates NLRP3 expression in a cerebral ischemia/reperfusion-induced neuronal injury rat model
}

\author{
Wei Li, Huisheng Chen, Zhijia Xu, Yan Lv, Ziai Zhao, Jinghua Zhang, Fang Qu \\ Department of Neurology, General Hospital of Northern Theater Command, Shenyang, Liaoning, China
}

\begin{abstract}
This investigation evaluated the neuroprotective effect of melodinhenine B in a cerebral ischemia/reperfusion (I/R)-induced neuronal injury rat model. The effect of melodinhenine $B$ was determined by evaluating the neurological deficit score, cerebral infarcted area, and blood-brain barrier (BBB) permeability. Moreover, the level of inflammatory cyto-

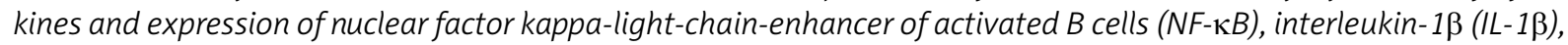
NLRP3, zonula occludens-1 (ZO-1), and occluding proteins were estimated by Western blotting. Histopathological changes and immunohistochemical analysis were performed to estimate the effect of melodinhenine $B$ on neuronal injury. The neurological deficit score, percentage of infarcted area, and $B B B$ permeability were improved in the melodinhenine $B$-treated group of rats. Treatment with melodinhenine $B$ attenuated the altered expression of $N F-\kappa B$, $I L-1 \beta, N L R P 3, Z O-1$, and occluding proteins in the brain tissue of I/R-induced neuronal injury rats. The inflammatory cytokine levels were reduced in the melodinhenine $B$-treated group. Histopathologically, melodinhenine $B$ reversed the pathological changes in the brain tissues of I/R-induced neuronal injury rats. In conclusion, melodinhenine $B$ protects against neuronal injury in cerebral ischemia-reperfusion injury rats by regulating the inflammasomes.
\end{abstract}

Key words: melodinhenine B, neuronal injury, ischemia-reperfusion, inflammasomes.

\section{Introduction}

Cerebral ischemia is a major cause of physical disability and mortality worldwide [6]. Reperfusion after the management of ischemia leads to secondary cerebral injury, commonly known as ischemia/reperfusion (I/R) injury. Several pathways are involved in the development of secondary injury, including autophagy, apoptosis, calcium overload, excitotoxicity, oxidative stress, impaired energy production, and inflammation [12]. The management of secondary injury due to cerebral I/R-induced neuronal injury has several limitations.
Autophagy in neuronal cells is regulated by the inflammatory cascade via inflammasomes [4]. Inflammation and immunity are regulated by inflammasomes, and NLRP3 is a major intracellular multiprotein complex that recognises pathogen-associated molecular patterns [11]. NLRP3 enhances the inflammatory response by activating cytokines and caspase-1 at the site of injury [1]. NLRP3 is involved in the development of disorders such as Alzheimer's disease, atherosclerosis, cancer, and diabetes [10]. Moreover NLRP3 is involved in the development of cerebral I/R-induced neuronal injury [16].

\section{Communicating author}

Wei Li, PhD, Department of Neurology, General Hospital of Northern Theater Command, No. 83, Wenhua Road, Shenhe District, Shenyang, Liaoning 110016, China, e-mail: NigelBoothogn@yahoo.com 
Several molecules from natural/herbal sources have proven roles in the treatment of chronic disorders. Traditionally, Melodinus henryi (Apocynaceae) has been used in China for treating bone fractures and meningitis [8]. Chemically, melodinhenine $B$ is an eburnean-vindolinine-type bisindole alkaloid isolated from $M$. henryi [15] which has strong anti-inflammatory and nitric oxide inhibitory activity $[2,15]$. Therefore, this study evaluated the neuroprotective effects of melodinhenine $B$ in a cerebral I/R-induced injury rat model.

\section{Material and methods}

\section{Animals}

Male Sprague-Dawley rats weighing 150-200 g were kept under a 12-h light/dark cycle at $60 \pm 5 \%$ humidity and $24 \pm 3^{\circ} \mathrm{C}$. The study protocols were approved by the animal ethics committee of the General Hospital of Northern Theater Command, China (IAEC/GHNTC/2017/09).

\section{Chemicals}

Melodinhenine B was supplied by the Laboratory of Natural Medicines, Nanjing, China. ELISA kits were procured from Thermo Fisher Scientific, China. Primary antibodies used in the Western blot assay were procured from Cell Signaling Technology (Beverly, MA, USA), and secondary antibodies from Tianjin Sungene Biotech (Tianjin, China).

\section{Experimental study}

Ischemia/reperfusion-induced neuronal injury was obtained using a middle cerebral artery occlusion model, as previously reported [3]. The left external and common carotid arteries were ligated after exposing them in anesthetised animals. A monofilament nylon suture was used to ligate the middle cerebral artery (MCA). The monofilament was inserted until it reached the origin of the MCA and local cerebral blood flow decreased to $<16 \%$ of the baseline level. Reperfusion involved restoring the cerebral blood flow to $75 \%$ after 1 hour of ischemia by releasing the ligature.

Animals were separated into three groups: the sham group underwent surgery without I/R induction; the I/R group underwent I/R; and the melodinhenine $B$ group received melodinhenine $B 100 \mathrm{mg} / \mathrm{kg}$ intraperitoneally at the time of reperfusion.

\section{Neurological dysfunction}

Neurological dysfunction was determined by neurological scores in I/R-induced neuronal injury rats using Longa's method [20]. The scoring was as follows: 0 - no deficit; 1 - forelimb failed to extend entirely and weak; 2 - circling to the contralateral side; 3 - weight-bearing capacity reduced on the injured side; and 4 - no spontaneous locomotor activity.

\section{Estimation of cerebral infarction}

The brain was isolated one day after reperfusion, and brain coronal slices were sectioned into 2-mm-thick sections and stained for $30 \mathrm{~min}$ at $37^{\circ} \mathrm{C}$ with $2 \%$ 2,3,5-triphenyltetrazolium chloride. Image J software was used to determine the percentage of the infarcted area in the slices from photographs of the slices.

\section{Estimation of blood-brain barrier permeability}

The blood-brain barrier (BBB) permeability was estimated by examining the extravasation of Evans Blue (EB) into the brain. Animals were injected with $2 \% \mathrm{~EB}$ at a dose of $2 \mathrm{ml} / \mathrm{kg}$ through the tail vein immediately after reperfusion. The brain was dissected from each animal after sacrifice, and the brain tissue was homogenised in phosphate-buffered saline. The supernatant of the homogenate was separated by centrifugation for $30 \mathrm{~min}$ at 15,000 rpm. Trichloroacetic acid solution (50\%) was used to dilute the supernatant, which was then incubated for $2 \mathrm{~h}$ at room temperature. A $160 \mathrm{~A}$ spectrophotometer was used to determine the absorbance of the supernatant at $610 \mathrm{~nm}$.

\section{Estimation of cytokines}

The levels of the inflammatory mediators interleukin (IL)-1 $\beta$, IL-6, and tumour necrosis factor $\alpha$ (TNF- $\alpha$ ) were determined in the brain tissue homogenate using enzyme-linked immunosorbent assays (ELISA) as per the kit protocols.

\section{Western blotting}

The nuclear factor kappa-light-chain-enhancer of activated $B$ cells (NF-kB), IL-1 $\beta$, NLRP3, zonula occludens-1 (ZO-1), and occluding proteins expressions were assessed using Western blot assays of 


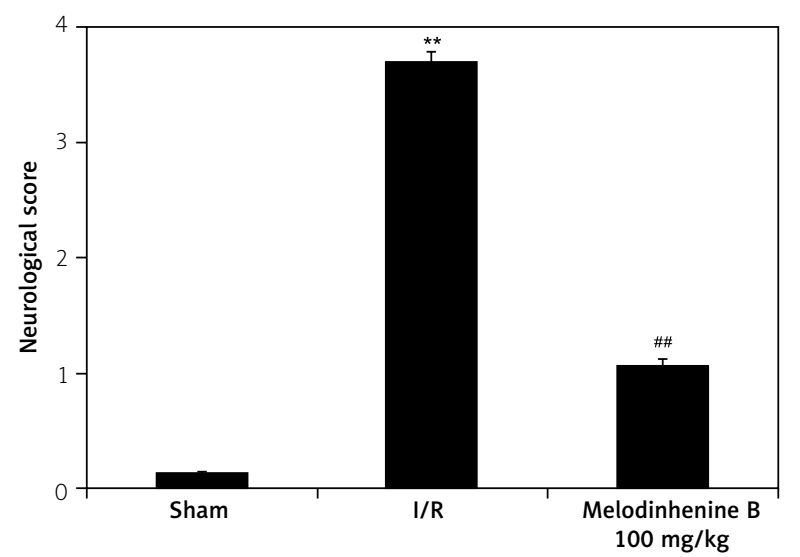

Fig. 1. Effect of melodinhenine $B$ on the neurological deficit score in an I/R-induced neuronal injury rat model. Mean \pm SEM $(n=10)$. ${ }^{* *} p<0.01$ compared with the sham group; ${ }^{\# \#} p<0.01 \mathrm{com}-$ pared with the I/R group.

brain tissue homogenates. A BCA assay kit was used to quantify the protein from each tissue homogenate, and $10 \%$ sodium dodecyl sulphate-polyacrylamide gel electrophoresis was used to separate the proteins, which were transferred to a nitrocellulose membrane by electroblotting. Subsequently, each membrane was blocked with $5 \%$ blocking solution (non-fat milk) and incubated in blocking buffer with primary antibodies overnight at $4^{\circ} \mathrm{C}$. Goat secondary antibodies conjugated with horseradish peroxidase were added to the blocking buffer, and a chemiluminescence kit was used to detect the proteins.

\section{Immunohistochemical analysis}

The 3,3'-diaminobenzidine method was used to stain the brain tissue immunohistochemically, as previously reported. Isolated brain tissue was sectioned and then incubated with NLRP3, ZO-1, or occluding protein antibodies at $4^{\circ} \mathrm{C}$ overnight. $\mathrm{A} B \times 51$ light microscope was used determine the immunoreactive proteins, and protein densities were estimated using Image-Pro Plus.

\section{Histopathological analysis}

Formaldehyde (4\%) was used to fix the brain tissues, which were embedded in paraffin, sectioned to $5-\mu \mathrm{m}$ thicknesses using a microtome, and stained with haematoxylin and eosin (H\&E). A trinocular microscope was used to evaluate pathophysiological changes in the brain tissue.

\section{Statistical analysis}

All data are expressed as mean \pm the standard error of the mean (SEM; $n=10)$. The statistical analysis consisted of one-way analysis of variance (ANOVA). The means were compared with Dunnett's post hoc test using GraphPad Prism (ver. 6.1; San Diego, CA, USA). $P$-values $<0.05$ were considered to indicate statistical significance.

\section{Results}

\section{Melodinhenine B improves the neurological function}

Figure 1 shows the effect of melodinhenine $B$ on the neurological deficit score in I/R-induced neuronal injury rats. The score was 3.7 in the $I / R$ group and 1.07 in the melodinhenine B-treated I/R-induced neuronal injury rats.

\section{Melodinhenine $B$ reduces the cerebral infarcted area}

The area of cerebral infarction was determined in the brains of I/R-induced neuronal injury rats (Fig. 2). The percentage area of cerebral infarction was $29 \%$ higher in the I/R group than in the shamoperated group, and it was reduced by $14 \%$ in the melodinhenine B-treated I/R-induced neuronal injury rat.

\section{Melodinhenine $B$ reduces blood-brain barrier permeability}

Using EB staining, the permeability of the BBB was observed in the cerebral tissues of I/R-induced neuronal injury rats treated with melodinhenine $B$. There was a significant increase in the EB staining in the cerebral tissue of the I/R group compared with the sham-operated group. Treatment with melodinhenine $B$ ameliorated the altered permeability of the $\mathrm{BBB}$ in the cerebral tissue of I/R-induced neuronal injury rats (Fig. 3).

\section{Melodinhenine $B$ reduces the levels of inflammatory mediators}

Figure 4 shows the levels of inflammatory cytokines in the brain tissue of I/R-induced neuronal injury rats. The TNF- $\alpha, \mathrm{IL}-1 \beta$, and IL- 6 levels were increased in the brain tissue homogenate of the I/R group compared with the sham-operated group. How- 


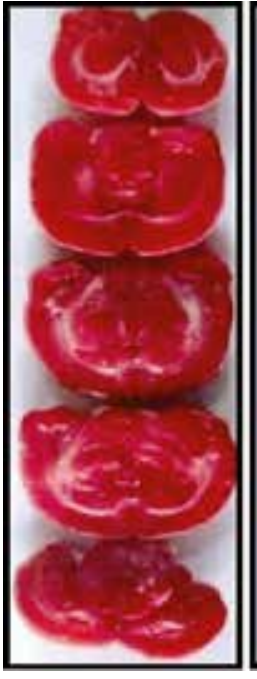

Sham

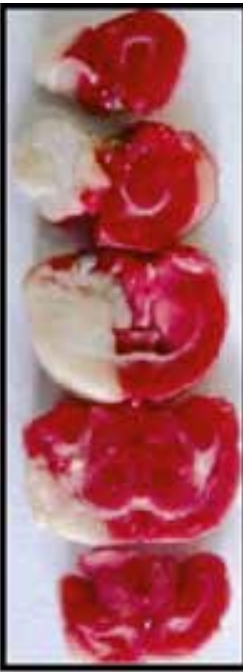

I/R

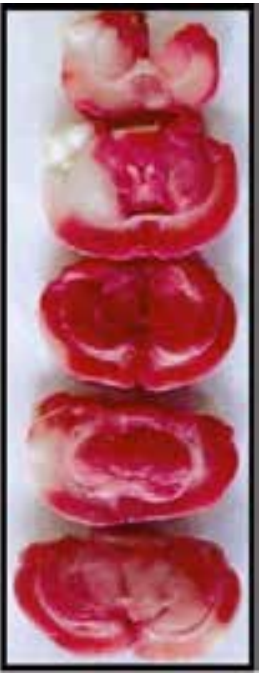

Melodinhenine B

Fig. 2. Effect of melodinhenine $B$ on the percentage of the cerebral infarct area in an I/R-induced neuronal injury rat model. Mean \pm SEM $(n=10)$. ${ }^{* *} p<0.01$ compared with the sham group; ${ }^{\# \#} p<0.01$ compared with the I/R group.

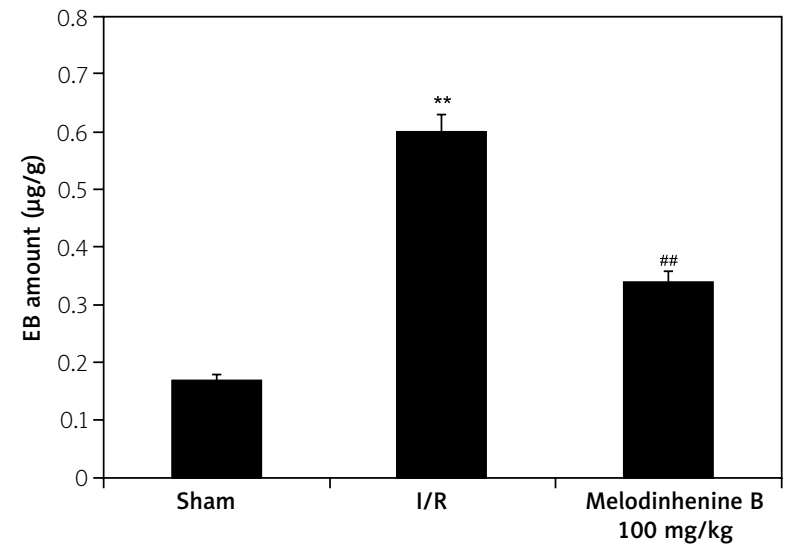

Fig. 3. Effect of melodinhenine B on BBB permeability in an I/R-induced neuronal injury rat model. Mean \pm SEM $(n=10) .{ }^{* *} p<0.01$ compared with the sham group; ${ }^{\# \#} p<0.01$ compared with the I/R group.

ever, there was a significant reduction in the cytokine levels in the brain tissue homogenate of the melodinhenine-B-treated group compared with the I/R group.

\section{Melodinhenine $B$ attenuates the expression of NF- $\kappa B$, IL-1 $\beta$, NLRP3, ZO-1, and occluding proteins}

Figure 5 shows the expression of $N F-\kappa B, I L-1 \beta$, NLRP3, ZO-1, and occluding proteins in melodin-

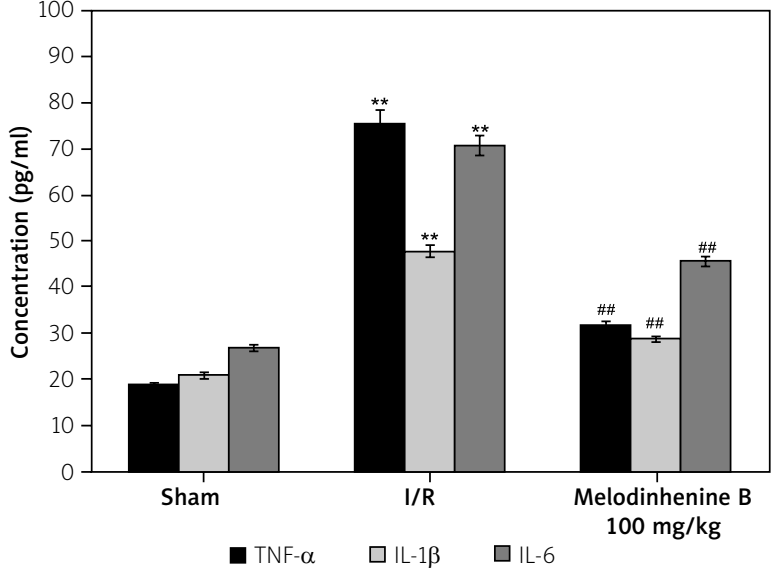

Fig. 4. Effect of melodinhenine $B$ on inflammatory mediators in an I/R-induced neuronal injury rat model. Mean $\pm \operatorname{SEM}(n=10) .{ }^{* *} p<0.01$ compared with the sham group; ${ }^{\# \#} p<0.01 \mathrm{com}-$ pared with the I/R group.

henine-B-treated I/R-induced neuronal injury rats by Western blotting. The expression of NLRP3, IL-1 $\beta$, and NF- $\kappa$ B proteins was enhanced and that of ZO-1 and occluding proteins was reduced in the brain tissues of the I/R group compared with the sham-operated group. However treatment with melodinhenine $B$ attenuated the expression of NF- $\mathrm{B}, \mathrm{IL}-1 \beta, \mathrm{NLRP} 3$, ZO-1, and occluding proteins in the I/R-induced neuronal injury rats. 

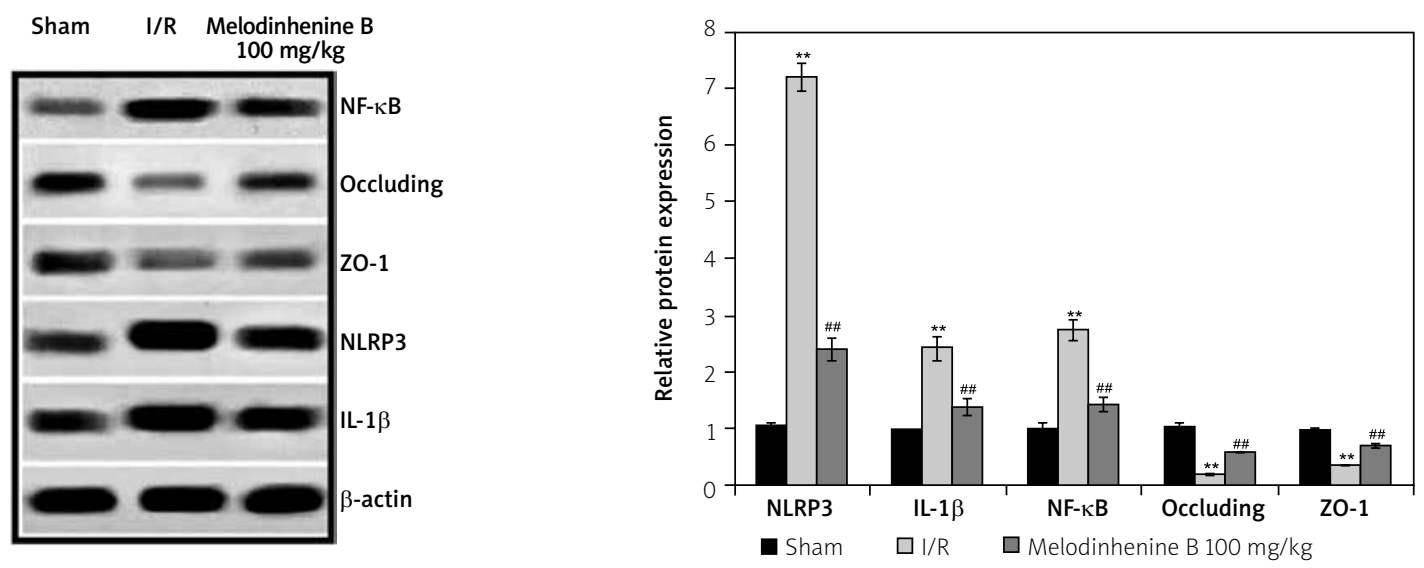

Fig. 5. Effect of melodinhenine $B$ on the expression of NF- $\kappa B, I L-1 \beta, N L R P 3, Z O-1$, and occluding proteins in an I/R-induced neuronal injury rat model by Western blot assay. Mean \pm SEM $(n=10) .{ }^{* *} p<0.01$ compared with the sham group; ${ }^{\# \#} p<0.01$ compared with the I/R group.

\section{Melodinhenine $B$ reduces the expression of NLRP3, ZO-1 and occluding proteins}

The expression of NLRP3, ZO-1, and occluding proteins in the brain tissue of melodinhenine-B-treated I/R-induced neuronal injury rats was determined immunohistochemically (Fig. 6). The expression of NLRP3 was enhanced, and that of ZO-1 and occluding proteins was reduced, in the brain tissue of I/R-induced neuronal injury rats. However, the expression of NLRP3 was reduced, and that of ZO-1 and occluding proteins was enhanced, in the brain tissue of the melodinhenine-B-treated group compared with the I/R group.

\section{Melodinhenine B ameliorates histopathological changes in the brain tissue}

Figure 7 shows the histopathological changes in the brain tissues of I/R-induced neuronal injury rats. Brain tissue sections of the sham-operated group appeared normal. The brain tissues of the I/R group showed nuclear and cytoplasmic vacuolation in the white matter and cortex. Moreover, the neurocytes were unconsolidated and disordered. However, treatment with melodinhenine $B$ ameliorated these pathological changes in the brain tissues of I/R-induced neuronal injury rats.

\section{Discussion}

Cerebral ischemia is a major cause of brain injury, and its management remains challenging. Therefore, we evaluated the protective effect of melodinhenine $B$ against I/R-induced neuronal injury by evaluating the neurological deficit score, cerebral infarcted area, and BBB permeability. In addition, the levels of inflammatory cytokines and expression of NF- $\kappa B$, IL-1 $\beta$, NLRP3, ZO-1, and occluding proteins were estimated by ELISA and Western blotting, respectively. Histopathological and immunohistochemical analyses were performed to examine the effect of melodinhenine B on neuronal injury.

I/R-induced cerebral injury leads to disruption of the BBB, which causes further secondary neuronal injury [17]. This suggests that ischemic brain disease progresses due to the linear relationship between cerebral I/R injury and disruption of the $B B B$ [19]. Maintaining the integrity of the BBB prevents neuronal injury and improves neurological function [18]. The permeability of the BBB was reduced in the melodinhenine-B-treated group compared with the I/R group. Cerebral ischemia leads to an increase in the area of infarcted brain, which also reduces the neurological function [13]. Based on the neurological deficit score and percentage of the infarcted area, melodinhenine- $B$ treatment improved the neurological function compared with the untreated I/R rats.

Tight junction proteins such as ZO-1 and occluding proteins contribute to maintaining the integrity of the BBB [5]. These proteins form a barrier that separates the cellular compartments and facilitates the exchange of ions and other substances. Altered ZO-1 and occluding protein levels may lead to chang- 


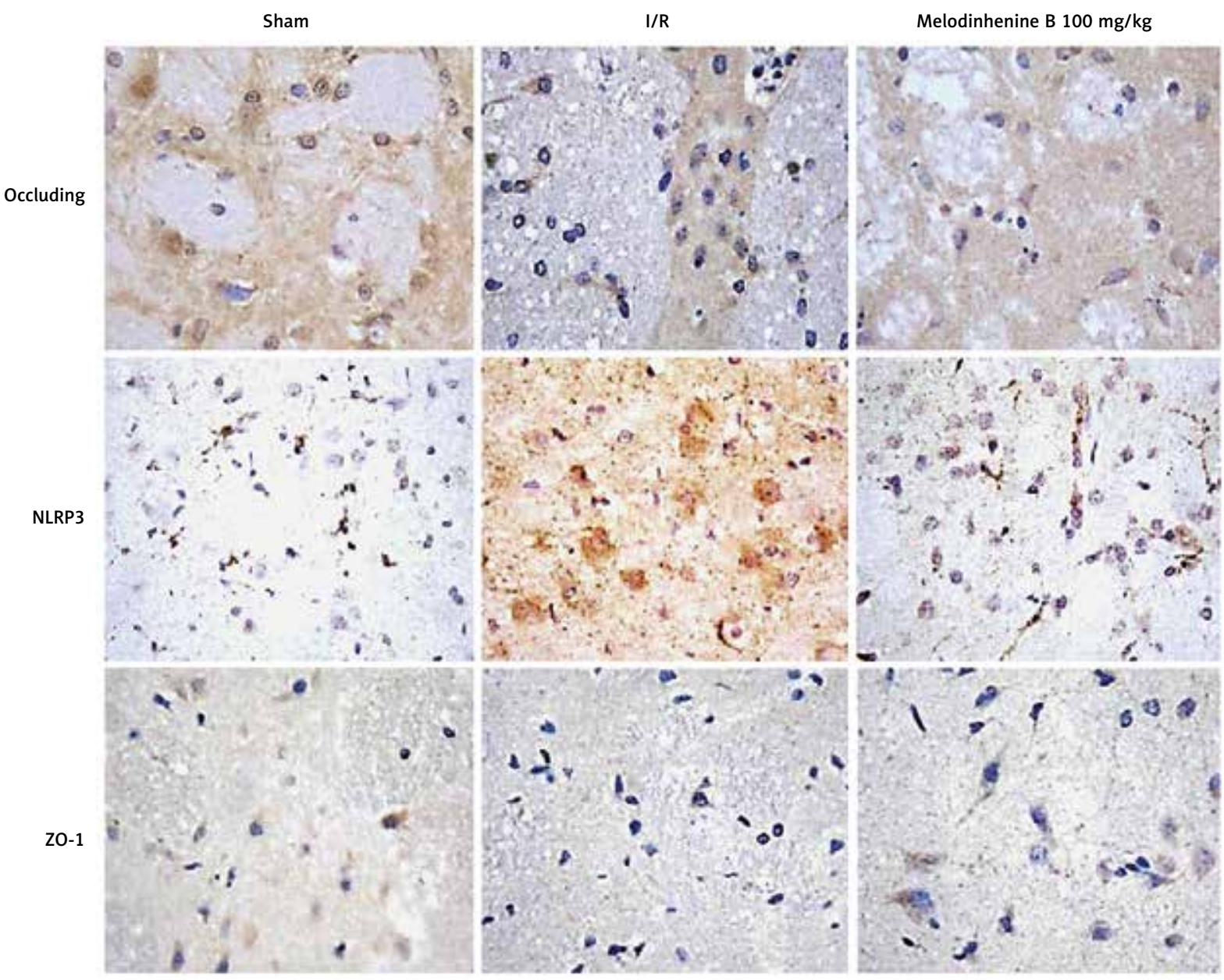

Fig. 6. Effect of melodinhenine $B$ on the expression of NLRP3, ZO-1, and occluding proteins in the brain tissue of an I/R-induced neuronal injury rat model by immunohistochemical analysis. Mean $\pm \operatorname{SEM}(n=10) .{ }^{* *} p<0.01$ compared with the sham group; ${ }^{\# \#} p<0.01$ compared with the I/R group.

es in the integrity of the BBB [9]. Tight junction proteins have a role in neuronal injury, and the expression of ZO-1 and occluding proteins protects against neuronal injury [23]. We found that treatment with melodinhenine $B$ normalized the altered expression of ZO-1 and occluding proteins in the cerebral tissues of I/R-induced neuronal injury rats.

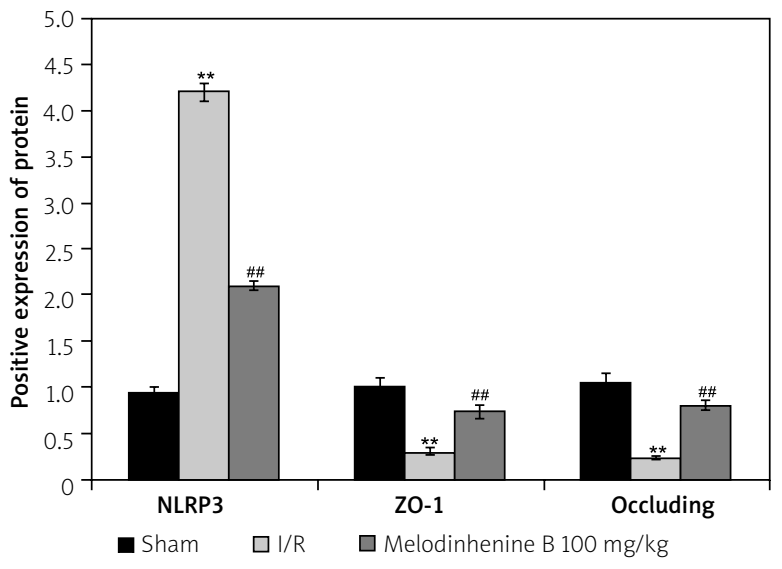

Inflammatory cytokines contribute to the development of I/R-induced neuronal injury [14]. The levels of these cytokines are enhanced with disruption of the BBB. The inflammatory response is enhanced by the activation of inflammasomes (NLRP3) in cerebral I/R-induced neuronal injury $[7,21]$. In cerebral disease, NLRP3 contributes to the disruption of the 


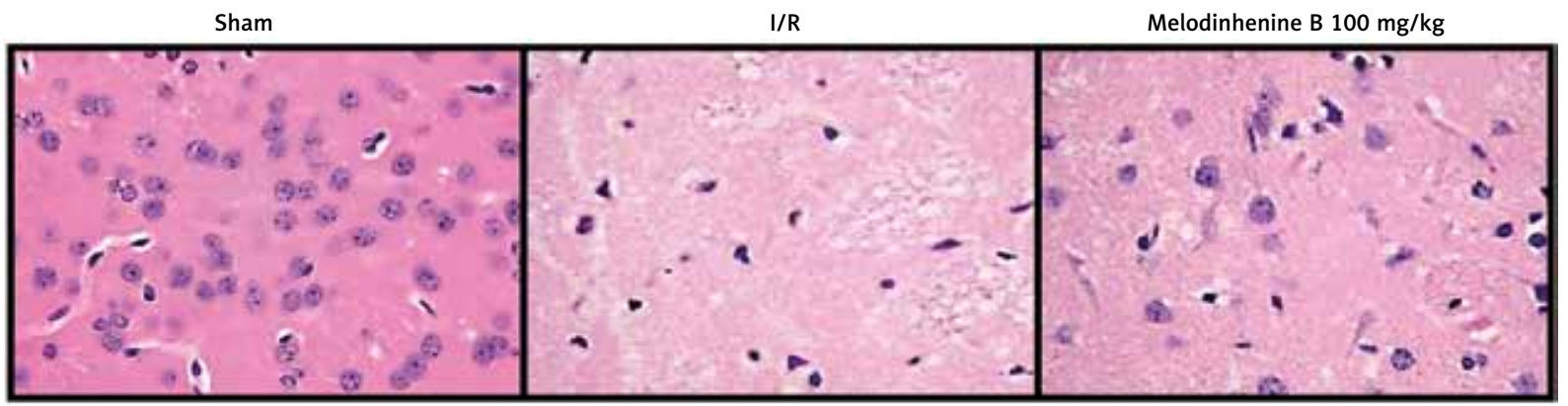

Fig. 7. Effect of melodinhenine B on the histopathological changes in the brain tissue of an I/R-induced neuronal injury rat model by $\mathrm{H} \& \mathrm{E}$ staining.

$B B B$, which leads to neuronal injury, whereas inhibition of NLRP3 prevents neuronal injury [22]. The expression of NLRP3 was reduced in the cerebral tissues of melodinhenine-B-treated I/R-induced neuronal injury rats.

\section{Conclusions}

Melodinhenine B protects against neuronal injury in cerebral ischemia-reperfusion injury rats by regulating the inflammasomes.

\section{Acknowledgments}

The authors thank the General Hospital of Northern Theater Command, China, for providing the facilities to conduct this work.

\section{Disclosure}

The authors report no conflict of interest.

\section{References}

1. An N, Gao Y, Si Z, Zhang H, Wang L, Tian C, Yuan M, Yang X, Li X, Shang $\mathrm{H}$, Xiong $\mathrm{X}$, Xing $\mathrm{Y}$. Regulatory mechanisms of the NLRP3 inflammasome, a novel immune-inflammatory marker in cardiovascular diseases. Front Immunol 2019; 10: 1592.

2. Arulselvan P, Fard MT, Tan WS, Gothai S, Fakurazi S, Norhaizan ME, Kumar SS. Role of antioxidants and natural products in inflammation. Oxid Med Cell Longev 2016; 2016: 5276130.

3. Bertrand L, Dygert L, Toborek M. Induction of ischemic stroke and ischemia-reperfusion in mice using the middle artery occlusion technique and visualization of infarct area. J Vis Exp 2017; 120: 54805.

4. Bussi C, Peralta Ramos JM, Arroyo DS, Gaviglio EA, Gallea J, Wang JM, Celej MS, Iribarren P. Autophagy downregulates pro-inflammatory mediators in BV2 microglial cells and rescues both LPS and alpha-synuclein induced neuronal cell death. Sci Rep 2017; 7: 43153.

5. Daneman R, Prat A. The blood-brain barrier. Cold Spring Harb Perspect Biol 2015; 7 :a020412.
6. Donkor ES. Stroke in the $21^{\text {st }}$ century: A snapshot of the burden, epidemiology, and quality of life. Stroke Res Treat 2018; 2018: 3238165.

7. Duan Q, Sun W, Yuan H, MuX. MicroRNA-135b-5p prevents oxygen-glucose deprivation and reoxygenation-induced neuronal injury through regulation of the GSK-3 $3 /$ Nrf2/ARE signaling pathway. Arch Med Sci 2018; 14: 735-744.

8. Feng T, Cai XH, Liu YP, Li Y, Wang YY, Luo XD. Melodinines A-G, monoterpenoid indole alkaloids from Melodinus henryi. J Nat Prod 2010; 73: 22-26.

9. Greene C, Campbell M. Tight junction modulation of the blood brain barrier: CNS delivery of small molecules. Tissue Barriers 2016; 4: e1138017.

10. Hong P, Gu RN, Li FX, Xiong XX, Liang WB, You ZJ, Zhang HF. NLRP3 inflammasome as a potential treatment in ischemic stroke concomitant with diabetes. J Neuroinflammation 2019; 16: 121.

11. Jo EK, Kim JK, Shin DM, Sasakawa C. Molecular mechanisms regulating NLRP3 inflammasome activation. Cell Mol Immunol 2016; 13: 148-159.

12. Kalogeris T, Baines CP, Krenz M, Korthuis RJ. Ischemia/reperfusion. Compr Physiol 2016; 7: 113-170.

13. Kawabori M, Yenari MA. Inflammatory responses in brain ischemia. Curr Med Chem 2015; 22: 1258-1277.

14. Lumniczky K, Szatmári T, Sáfrány G. Ionizing radiation-induced immune and inflammatory reactions in the brain. Front Immunol 2017; 8: 517.

15. Ma K, Wang JS, Luo J, Kong LY. Six new alkaloids from Melodinus henryi. Fitoterapia 2015; 100: 133-138.

16. Minutoli L, Puzzolo D, Rinaldi M, Irrera N, Marini H, Arcoraci V, Bitto A, Crea G, Pisani A, Squadrito F, Trichilo V, Bruschetta D, Micali A, Altavilla D. ROS-mediated NLRP3 inflammasome activation in brain, heart, kidney, and testis ischemia/reperfusion injury. Oxid Med Cell Longev 2016; 2016: 2183026.

17. Price L, Wilson C, Grant G. Blood-brain barrier pathophysiology following traumatic brain injury. In: Laskowitz D, Grant G (eds.). Translational Research in Traumatic Brain Injury. CRC Press/ Taylor and Francis Group, Boca Raton (FL) 2016.

18. Ronaldson PT, Davis TP. Blood-brain barrier integrity and glial support: mechanisms that can be targeted for novel therapeutic approaches in stroke. Curr Pharm Des 2012; 18: 3624-3644. 
19. Venkat P, Chopp M, Chen J. Blood-brain barrier disruption, vascular impairment, and ischemia/reperfusion damage in diabetic stroke. J Am Heart Assoc 2017; 6: e005819.

20. Vital SA, Gavins FN. Surgical approach for middle cerebral artery occlusion and reperfusion induced stroke in mice. J Vis Exp 2016; 116: 54302

21. Voet S, Srinivasan S, Lamkanfi M, van Loo G. Inflammasomes in neuroinflammatory and neurodegenerative diseases. EMBO Mol Med 2019; 11: e10248.

22. Xu B, He X, Sui Y, Wang X, Wang X, Ren L, Zhai YX. Ginkgetin aglycone attenuates neuroinflammation and neuronal injury in rats with ischemic stroke by modulating STAT3/JAK2/SIRT1. Folia Neuropathol 2019; 57: 16-23.

23. Ye ZY, Xing HY, Wang B, Liu M, Lv PY. DL-3-n-Butylphthalide protects the blood-brain barrier against ischemia/hypoxia injury via upregulation of tight junction proteins. Chin Med J (Engl) 2019; 132: 1344-1353. 